\title{
Patrimoines du Sud
}

$10 \mid 2019$

Châteaux, palais et tours : pouvoirs et cultures dans l'Occitanie médiévale

\section{Une fortification de terre languedocienne d'ampleur exceptionnelle, la motte du castrum de Mauguio (Hérault)}
A Languedoc fortification of exceptional size, the motte-and-bailey castle of
Mauguio

\section{Claude Raynaud}

\section{(2) OpenEdition}

\section{Journals}

\section{Édition électronique}

URL : http://journals.openedition.org/pds/2918

DOI : $10.4000 /$ pds.2918

ISSN : 2494-2782

\section{Éditeur}

Conseil régional Occitanie

\section{Référence électronique}

Claude Raynaud, « Une fortification de terre languedocienne d'ampleur exceptionnelle, la motte du castrum de Mauguio (Hérault) », Patrimoines du Sud [En ligne], 10 | 2019, mis en ligne le 02 septembre 2019, consulté le 10 décembre 2020. URL : http://journals.openedition.org/pds/2918 ; DOI : https:// doi.org/10.4000/pds.2918

Ce document a été généré automatiquement le 10 décembre 2020.

La revue Patrimoines du Sud est mise à disposition selon les termes de la Licence Creative Commons Attribution - Pas d'Utilisation Commerciale - Pas de Modification 4.0 International. 


\title{
Une fortification de terre
} languedocienne d'ampleur exceptionnelle, la motte du castrum de Mauguio (Hérault)

\author{
A Languedoc fortification of exceptional size, the motte-and-bailey castle of \\ Mauguio
}

Claude Raynaud

1 Au centre de la ville de Mauguio, le jardin municipal de la Motte occupe un site majeur du patrimoine médiéval languedocien. C'est là que la puissante famille de Melgueil établit un château vers l'an Mil, organe du pouvoir comtal, qui donna plusieurs évêques à Maguelone. C'est aussi là que fut frappée jusqu'à la fin du Moyen Âge l'une des principales monnaies du Midi médiéval, reprise ensuite par la monnaie de Montpellier (fig. 1). 
Fig. 1

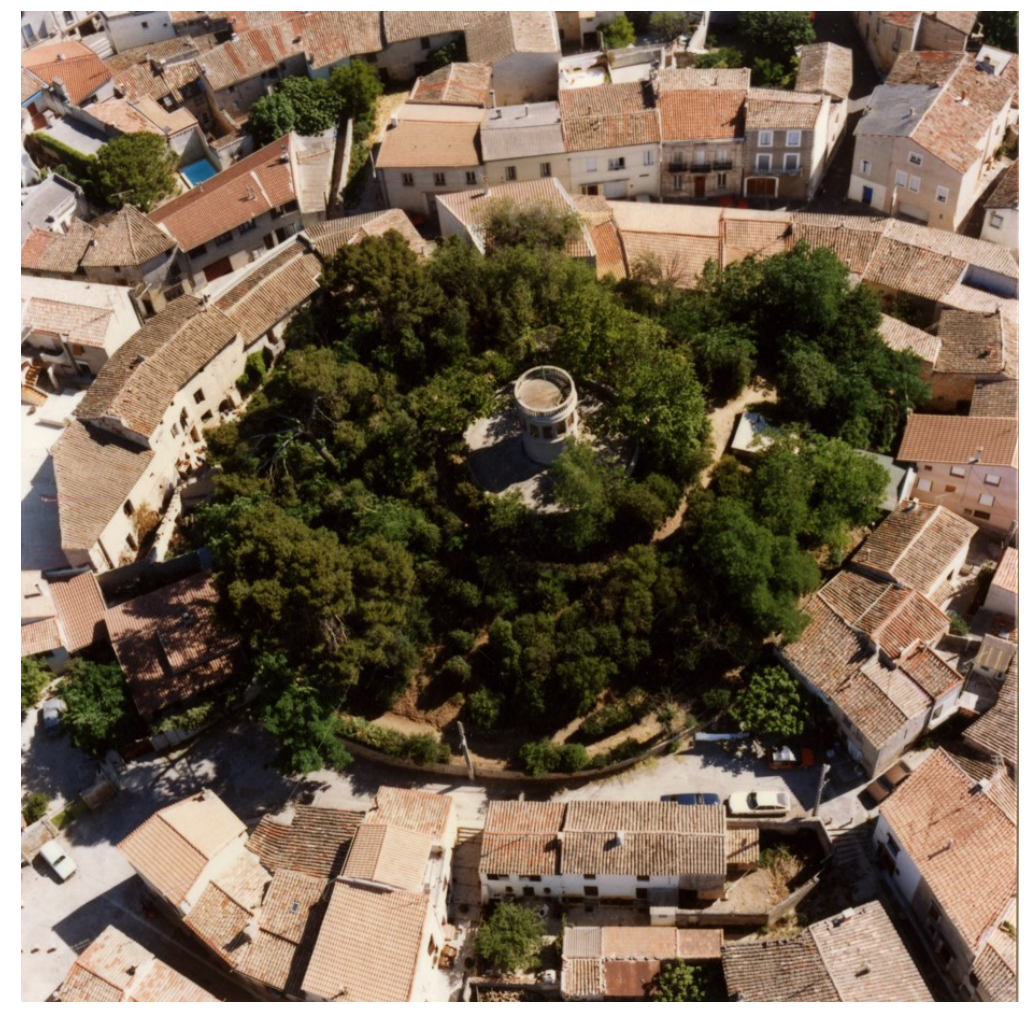

Mauguio (Hérault), vue aérienne du centre autour de la motte en 1988, avant la rénovation du jardin M. Baron ( A Atelier d'Archéologie Lunel-Viel

2 Enserré dans des constructions modernes, resté à l'abandon durant de longues décennies, ce monument a fait l'objet d'un réaménagement au début du XXI ${ }^{\mathrm{e}}$ siècle. Préalablement aux travaux de réhabilitation du site, une étude archéologique a été réalisée en 1989-1991 afin d'analyser la structure initiale et la morphologie de la Motte, d'en dater la création et de la replacer dans le développement topographique du bourg castral.

3 Au pied sud de la motte, l'îlot circonscrit par les rues Diderot et Gambetta ainsi que par la place Jean Jaurès, est traditionnellement identifié comme ayant abrité le château comtal du second Moyen Âge. Divers éléments architecturaux, aujourd'hui démembrés entre plusieurs habitations, témoignent en effet de la présence d'une demeure aristocratique, objet de multiples transformations du XVI ${ }^{e}$ au XXe siècle. Acquis par la commune de Mauguio, restauré et réhabilité, l'un des édifices a permis de réaliser en 2012 et 2014 une étude archéologique sur l'occupation des lieux antérieurement à la construction des bâtiments, tout en complétant le dossier sur l'origine de la motte castrale et son devenir. Ces investigations éclairent l'émergence d'un castrum, le cadre de la genèse urbaine et plus largement l'incastellamento de la plaine montpelliéraine.

\section{Historiographie d'un château méconnu}

«Mauguio ou Melgueil est sur l'étang et communique avec la mer, comme Maguelone, par un grau. Au centre de la ville est une éminence, en terre rapportée, couronnée par un moulin à vent, et d'où la vue s'étend au loin. On pense qu'elle a dû être faite pour servir d'observatoire ou pour y construire un château. «Qui veut 
aller sur ceste montaigne, dit un proverbe du pays, fault qu'il monte un grand faix de terre $»^{1}$.

Le 13 juin 1598, Thomas Platter pose un regard éclairé sur la motte de Mauguio dont il nous offre la première description. Singularité du lieu, obscurité de son histoire et perplexité de l'observateur: archéologue avant l'heure, Platter brosse en quelques lignes le profil d'un château disparu. Sitôt aperçu, notre château sombre à nouveau dans l'oubli.

"Il ne subsiste plus rien du château de Mauguio, dont l'existence est constatée dès la fin du $\mathrm{X}^{\mathrm{e}}$ siècle. Mais, au centre du village, s'élève une motte féodale qui remonte vraisemblablement à cette époque. On y a découvert récemment un puits qui servait probablement, en temps de siège, à alimenter les défenseurs et les habitants réfugiés dans le donjon ou l'enceinte fortifiée, qui devait couronner cette hauteur. Nous ne connaissons aucun autre exemple de motte féodale dans la région ».

Par ces lignes allusives, Émile Bonnet rend compte en 1905 des découvertes de Joseph Berthelé, talentueux archiviste et archéologue des campagnes montpelliéraines. Berthelé et Bonnet demeurèrent longtemps les seuls historiens de la motte melgorienne ${ }^{2}$.

6 Après avoir été « inventée » sans grande démonstration au début du siècle, la motte faillit passer aux oubliettes de façon tout aussi abrupte. On retrouve en effet la motte en 1987 dans une synthèse sur les recherches autour de l'An Mil, avec une mention encore plus brève et sans l'ombre d'un argument: «É. Bonnet n'attribue ensuite au début des temps romans qu'un seul vestige d'architecture militaire : ce que J. Berthelé déclare être une motte féodale, à savoir la butte de Mauguio $»^{3}$.

7 Faute d'avoir bénéficié d'une étude solidement argumentée, la motte de Mauguio n'a pas manqué de stimuler l'imaginaire local, faisant naitre un récit qui tentait d'éclairer l'origine et la fonction du site. Une première version, sur un versant mythologique, voyait à l'origine l'emplacement d'un temple à Mercure qui, par évolution phonétique, Mercurium = Melgorium (!) aurait donné son nom au castrum. Défendue par le chanoine Paul Gariel au XVII ${ }^{e}$ siècle, cette thèse pseudo-étymologique fut fermement combattue au XIX ${ }^{e}$ siècle par André Germain et semble oubliée depuis lors ${ }^{4}$. Selon une version plus prosaïque, la butte de la motte correspondrait à l'accumulation des déblais extraits lors du creusement des fossés de la grande enceinte du bas Moyen Âge. Tout aussi infondée que la première, cette hypothèse supposait un bel effort d'anticipation consistant à réserver, au $\mathrm{XI}^{\mathrm{e}}$ siècle dès l'émergence du castrum, un vaste espace vacant au centre de la ville, afin d'y déposer les déblais quatre siècles plus tard !

De façon plus convaincante, la tradition locale a conservé le souvenir d'un rite d'intégration des jeunes hommes ou des nouveaux habitants, qui devaient pour être acceptés transporter un sac de terre jusqu'au sommet de la motte pour l'y déverser. Ainsi se perpétua jusqu'au début du $\mathrm{XX}^{\mathrm{e}}$ siècle, sur le mode symbolique, le geste des paysans médiévaux qui édifièrent la butte : nous voilà revenus à la version de Platter, à l'évidence informée par la même tradition.

9 Perforée par un château d'eau au début du $\mathrm{XX}^{\mathrm{e}}$ siècle, puis enfouie sous la végétation d'un jardin public, la motte cacha longtemps encore son potentiel archéologique. Dégradée par l'érosion et fermée au public pour raison de sécurité, la motte devenait inaccessible, jusqu'à ce qu'un projet de réaménagement du jardin donne l'opportunité d'y effectuer de premiers sondages en 1989-1990. De nouveaux sondages purent être réalisés en 2012-2014, à l'occasion de la réhabilitation d'une partie du «château », au 
pied sud de la motte ${ }^{5}$. Bien que d'étendue limitée par le contexte urbain ainsi que par la fragilité du site, à vingt-cinq ans d'intervalle ces sondages ont livré des données décisives confirmant l'origine artificielle et la fonction castrale de la motte de Mauguio.

\section{La motte aux origines du castrum}

Le castrum melgorium est mentionné pour la première fois dans un acte du cartulaire de l'abbaye de Gellone, à une date incertaine entre les années 996 et 1031. Il est localisé in comitatu Substantionense, in vigaria Magdalonense $e^{6}$. À $15 \mathrm{~km}$ au nord-est du siège épiscopal de Maguelone, $10 \mathrm{~km}$ au sud-est du castrum comtal de Substantion (Castelnau-le-Lez) et à seulement $2,5 \mathrm{~km}$ du littoral de la lagune littorale, le site de Mauguio revêt une valeur stratégique au cœur de la plaine, pour le contrôle des communications terrestres et lagunaires. L'intérêt de cette position fut probablement accentué dans le contexte des incursions arabes qui ravagèrent le littoral au cours des $\mathrm{IX}^{\mathrm{e}}-\mathrm{X}^{\mathrm{e}}$ siècles.

Les sources textuelles médiévales mentionnant le castrum de Mauguio restent allusives et ne font jamais état d'une motte, silence qui ne doit pas surprendre dans des actes comparables qui décrivent bien rarement la matérialité et l'organisation du castrum. Moins habituelle est la représentation figurée qui apparaît sur une carte de l'étang de Mauguio dressée au milieu du XIV ${ }^{e}$ siècle pour le contrôle de la pêche et des eaux entre Maguelone et Mauguio ${ }^{7}$ (fig. 2).

Fig. 2

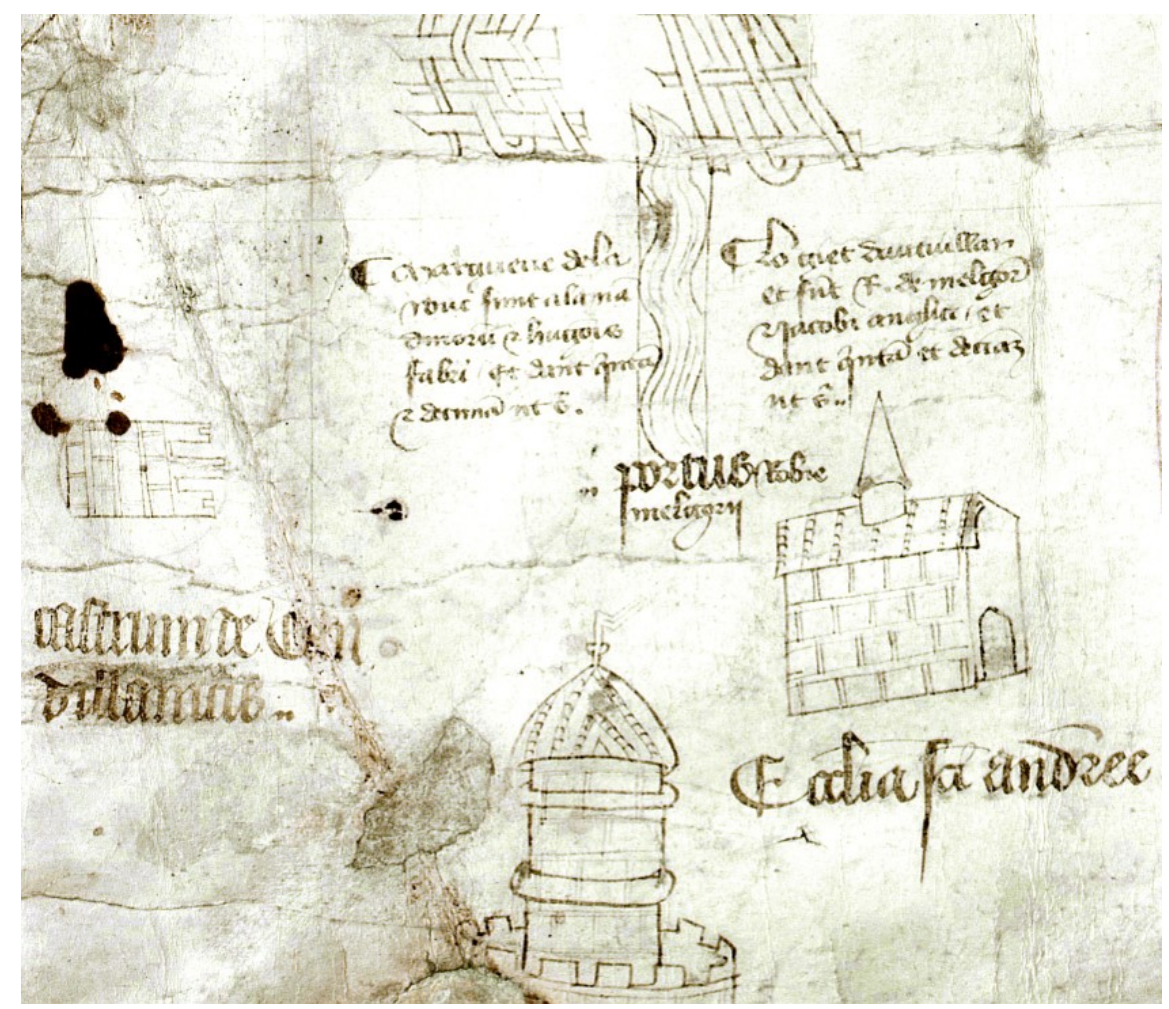

Mauguio (Hérault), localisation du castrum sur la carte des pêcheries de l'étang au XIVe siècle. Archives départementales de l'Hérault, G 2046-1, (détail).

(C) AD Hérault 
D'un dessin précis et juste topographiquement, la carte sur parchemin permet de localiser dans l'angle bas à gauche le castrum Candillanicis, aujourd'hui Candillargues, et vers la droite l'église Saint-André de Granolhers, aujourd'hui disparue mais localisée sur des plans anciens. Entre les deux, légèrement au nord (le nord est figuré vers le bas), Mauguio est aisément identifiable grâce au dessin d'une grosse tour ronde crènelée, au-dessus de laquelle apparaît l'emplacement du portus robi(n)e melgorii, le port de la Robine de Mauguio, roubine toujours localisée sur les cartes actuelles ${ }^{8}$.

Malgré son intérêt topographique et sa richesse documentaire sur la gestion de l'étang, la carte ne fait nulle mention ni allusion graphique à la présence de la motte qui porterait la tour, le dessin étant rogné au bord du parchemin. Que signifie l'absence de la motte sur un tel document : omission ou état de fait? La carte n'avait probablement pas à porter un tel détail, mais on verra qu'en effet, la butte paraît délaissée avant la fin du Moyen Âge. Elle est encore mentionnée sur des plans terrier du XVIII ${ }^{e}$ siècle, non comme élément défensif mais comme un point de repère ou portant un calvaire ou un édicule, un amer dans la platitude du paysage melgorien, repère probablement apprécié des marins qui l'apercevaient au loin (fig. 3).

Fig. 3

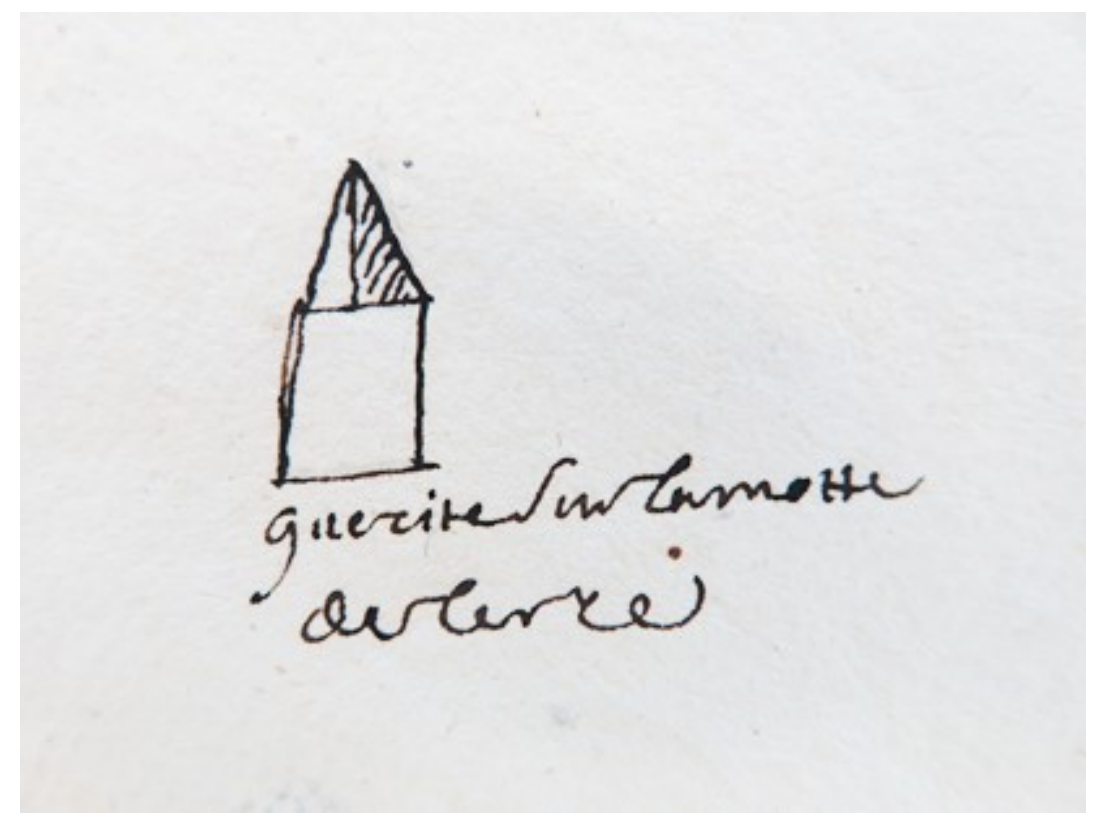

Mauguio (Hérault), « guérite sur la motte de terre », plan terrier du XVIIIe siècle. Archives départementales de l'Hérault, G 4443

(c) AD Hérault

Démunie de toute fonction militaire, la motte conservait cependant une certaine valeur économique en offrant dans la plaine le seul relief susceptible de porter un moulin à vent, qui apparaît en effet sur le plan cadastral de 1818 puis sur un dessin de 1835 (fig. 4). 
Fig. 4

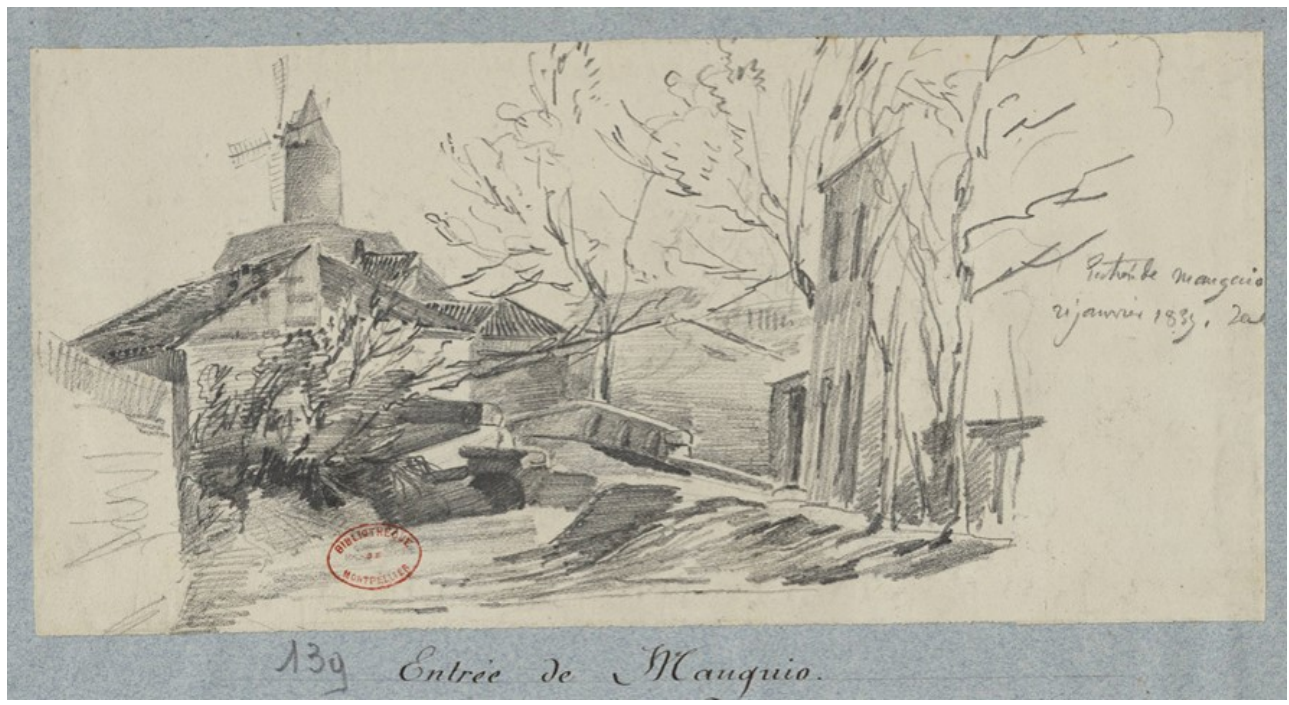

Mauguio (Hérault), dessin de Jean-Marie Amelin montrant le moulin sur la motte en 1835. Archives municipales de Montpellier, 1652RES-Vol 6-139

(c) AM Montpellier

Le moulin apparaît sans ailes ni toiture à la fin de ce siècle, mais le vent souffle toujours et bénéficie à l'installation, en 1904, d'un château d'eau et d'une éolienne chargée du pompage de l'eau municipale. La butte revêt alors l'essentiel de sa physionomie actuelle, à l'exception de l'éolienne, abattue par une tempête en 1907 (fig. 5). 
Fig. 5

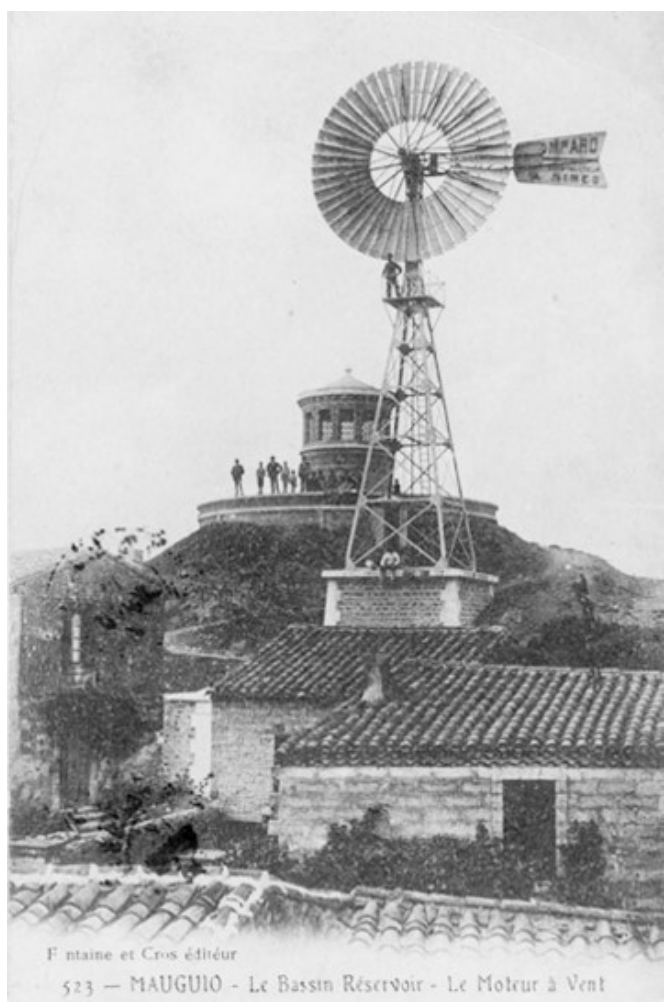

Mauguio (Hérault), photo de la motte au début du XXe siècle montrant l'éolienne et le réservoir du service des eaux. Observer la forte érosion des flancs de la butte et comparer l'édifice du réservoir avec la tour de la figure 4

(c) coll. particulière

\section{Première approche topographique}

Les photographies aériennes ainsi que les plans cadastraux soulignent le rôle initiateur de la motte dans la formation du castrum puis du bourg moderne dont elle occupe l'exact centre géométrique, conditionnant les lignes directrices de la topographie. En effet, l'analyse morphologique du tissu urbain permet d'identifier deux enveloppes circulaires correspondant probablement à des enceintes successives développées de façon concentrique autour de la motte (fig. 6). Selon cette lecture, l'enceinte première devait se situer sous les actuelles rues de la Motte et de La Bruyère qui enserrent le jardin de la Motte. Une seconde auréole, composée des rues Rousseau, Voltaire, du Quatre Septembre, Chénier, Gambetta et de la Monnaie, se développe plus largement tout en reproduisant la même forme circulaire. La figure circulaire du plan ne sera délaissée qu'à l'issue d'une nouvelle et radicale transformation qui voit le bâti s'étendre fort sensiblement puis s'enfermer dans une grande enceinte quadrangulaire, dès le second Moyen Âge. 
Fig. 6

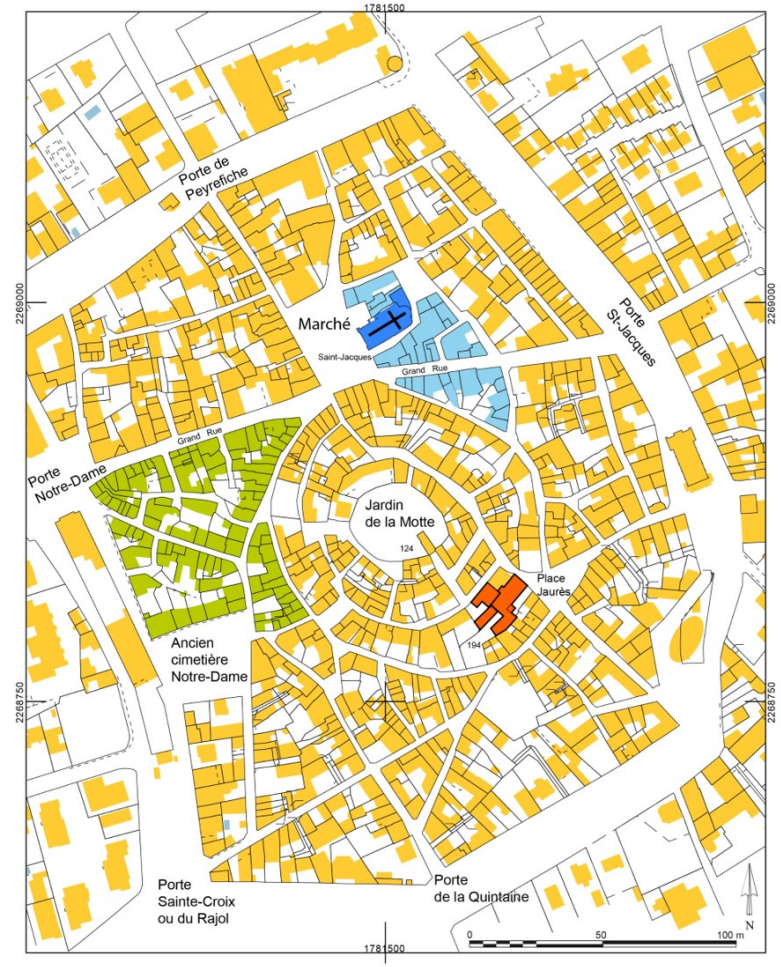

Mauguio (Hérault), plan parcellaire concentrique du centre ancien organisé autour de la motte et encadré par l'enceinte quadrangulaire du bas Moyen Âge. Les sondages archéologiques ont été réalisés dans le jardin de la motte et dans les parcelles en rouge, plan cadastral

DAO C. Raynaud (c) CNRS

17 Le modelé du terrain apparaît tout aussi éloquent : tandis que l'ensemble du bourg se déploie sur une topographie plane entre $6 \mathrm{~m}$ et 7,50 $\mathrm{m}$ d'altitude NGF, la motte culmine à $23 \mathrm{~m}$ et forme un point fort du paysage. Son emplacement est tout autant remarquable dans la plaine littorale, à l'intersection du camin salinié, chemin du sel d'origine antique, et du cours ancien de la Capouillère, cours d'eau canalisé vers les fossés de la ville médiévale mais dont le chenal fossile reste visible sur les cartes géologique et pédologique.

18 La formation de la motte ne pouvant recevoir une explication géomorphologique dans le contexte de la plaine littorale, l'hypothèse d'un relief artificiel, créé pour y établir un château, restait à établir. La démonstration fut aisée: en 1989, des carottages et sondages pressiométriques effectués préalablement aux travaux de confortement de la butte, révélèrent la présence d'un matériau de remblai, peu compact, d'origine alluviale ou colluviale, sur une épaisseur de 7 à $10 \mathrm{~m}$ sous le sol actuel. En 1990, un sondage archéologique conduit jusqu'à $1,9 \mathrm{~m}$ sous le sol du jardin, à mi pente de la butte, précisa ces observations en révélant l'existence d'importantes couches de remblai. Tous les sondages pratiqués en divers points de la motte montrèrent la même composition sédimentaire de limons argileux rapportés, d'origine diverse. D'après les observations pédologiques, ces remblais proviendraient, pour les couches grises, de prélèvements massifs dans les zones palustres entourant l'étang de l'or, et pour les couches de sédiment brun d'emprunts dans la plaine autour de Mauguio. 
Par la suite, un relevé topographique aux abords de la motte ainsi que des travaux de voirie dans les rues voisines, permirent d'observer des coupes de terrain. L'extension de la butte artificielle se confirma sous la rue de la Motte, où le paléosol ne fut jamais atteint par les excavations, un épais remblai de $0,75 \mathrm{~m}$ au minimum, de même nature que celui de la motte, étant visible jusqu'au fond des tranchées. Ces diverses observations convergeaient vers le même constat: au minimum, le relief artificiel s'étendait sur une centaine de mètres de diamètre, couvrant une large partie du «premier cercle» identifié dans le centre urbain. Il fallait par ailleurs restituer à la butte un profil plus accusé que l'actuel puisque le sommet de la butte témoignait d'une forte érosion, tandis que les remblais modernes s'accumulaient au pied de la motte, estompant le relief d'origine (fig. 7).

Fig. 7

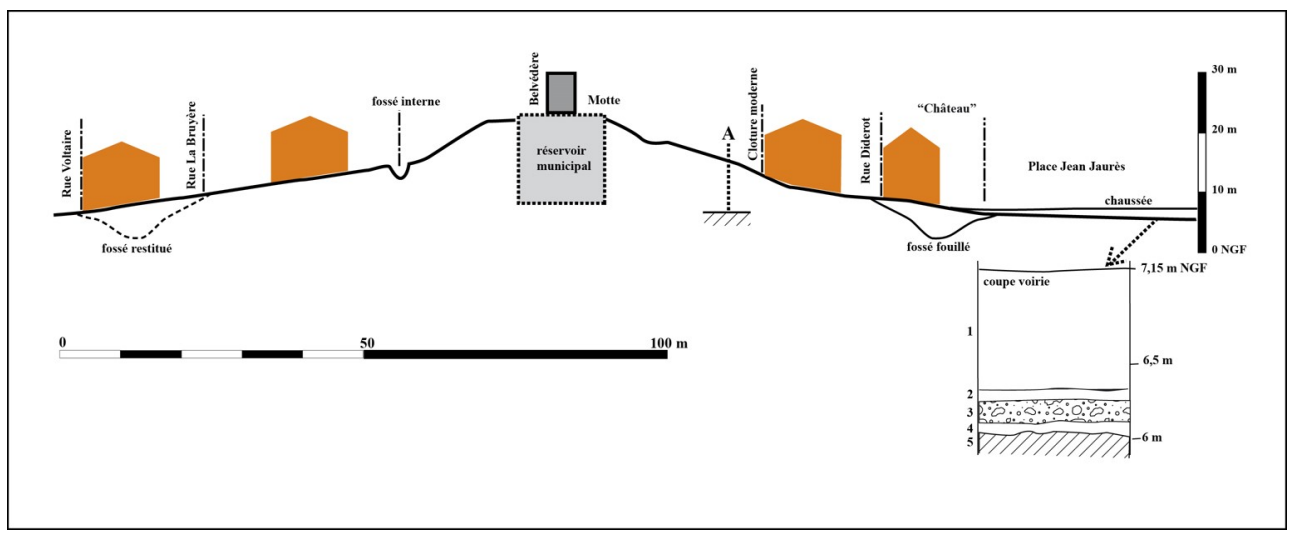

Mauguio (Hérault), coupe synthétique de la motte et de ses abords. A : sondage sédimentologique et pressiométrique indiquant 7 à 10 mètres d'épaisseur de terre rapportée. Vignette " coupe voirie »: 1. Sol actuel et remblais modernes. 2 : limon colluvial. 3 : sol de calade. 4 : paléosol anté-médiéval. 5 : substrat de «taparas »

Cl. Raynaud (c) CNRS

20 Partant de ces observations et de la topographie du centre de Mauguio on pouvait aussi envisager, ceinturant la motte, une basse-cour légèrement surélevée. Le profil de la butte révélait en effet, entre la clôture actuelle du jardin municipal et le passage de cette enceinte initiale, un relief moins accusé que dans la partie centrale. Cette interprétation présentait aussi l'intérêt de réduire le diamètre de la motte autour de $65 \mathrm{~m}$, taille moins surprenante avec tout de même une surface de 0,34 hectares. Le relief artificiel de la motte et de ses aménagements périphériques présentait donc une taille imposante par rapport aux dimensions courantes des mottes castrales, qu'elles soient d'origine naturelle ou artificielle.

\section{Aménagement d'une motte languedocienne}

21 Après les sondages de reconnaissance conduits en 1989, une aire de fouille de près de 200 mètres carrés fut ouverte en 1990 sur le flanc ouest de la butte (fig. 8). 
Fig. 8

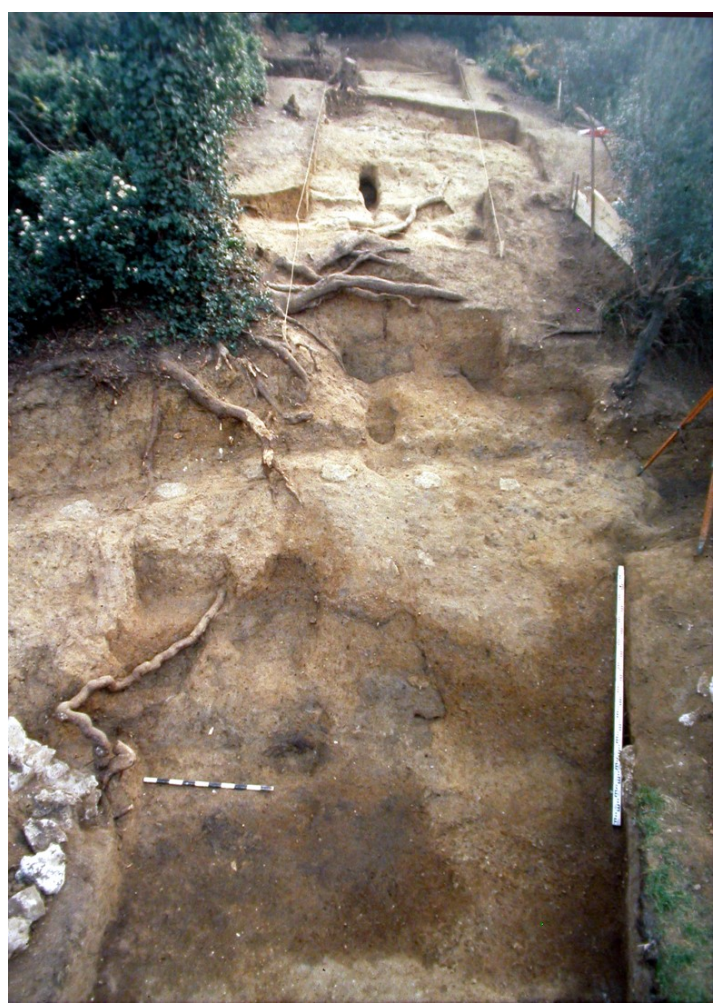

Mauguio (Hérault), vue de la fouille du flanc ouest (1990) révélant l'implantation de deux rangs de poteaux de palissade (sédiment gris sur fond beige) ; l'érosion des vestiges est aggravée par le foisonnement des racines

Cl. Raynaud (C) CNRS

22 Il s'agissait alors d'exhumer les vestiges d'aménagement de la motte et de tenter une datation. D'emblée s'imposa le constat d'une forte érosion de la butte dont le sol artificiel et le relief accusé ne conservaient que peu de traces des aménagements médiévaux. Sous les murets et les remblais modernes du jardin, subsistait néanmoins une série de profonds trous de poteaux dessinant l'emprise de deux lignes de palissades, une ligne basse à l'altitude de $16 \mathrm{~m}$, une autre à $19 \mathrm{~m}$ (fig. 9, 31, 32), tandis qu'au sommet se dessinait, conservée sur moins de deux mètres autour du réservoir moderne, une plateforme creusée de plusieurs fosses, parmi lesquelles un silo destiné au stockage de récoltes (id., 10, 42-43). 
Fig. 9

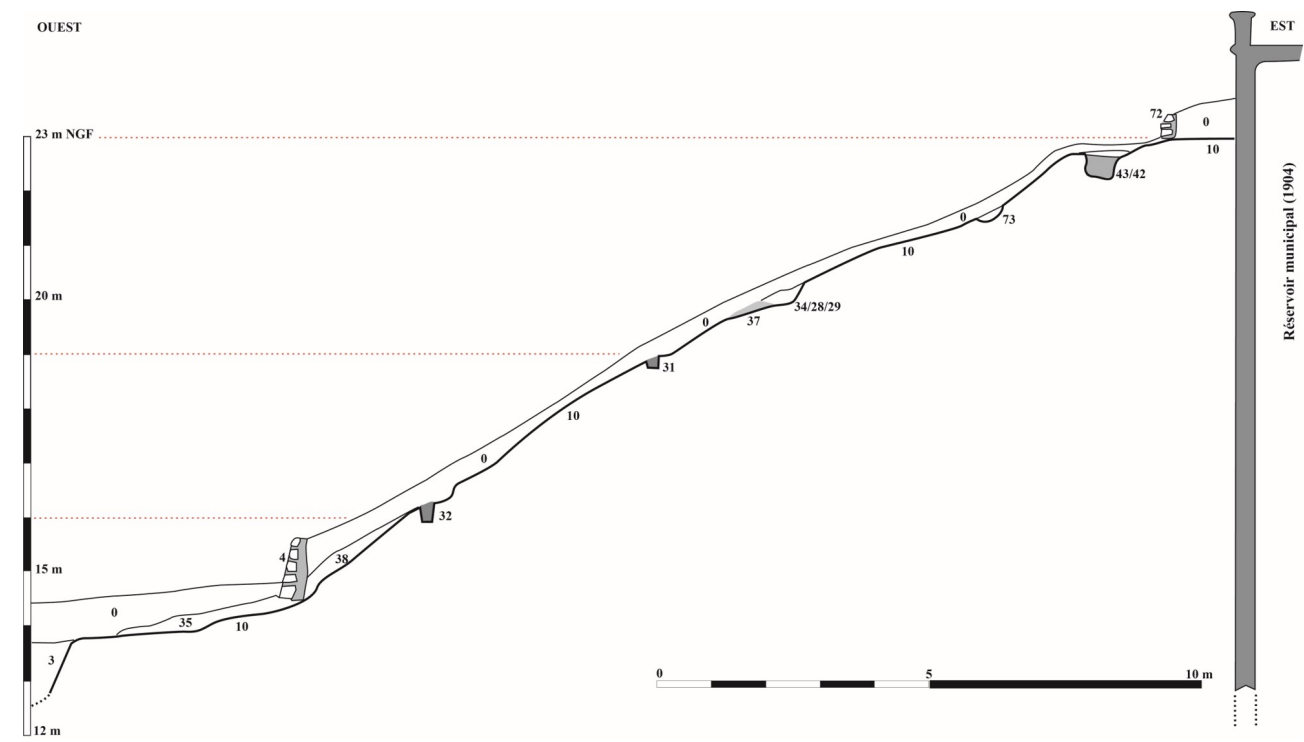

Mauguio (Hérault), coupe du flanc ouest de la motte d'après la fouille de 1990. En trait gras profil médiéval érodé, mettant en évidence les trous de poteaux de deux palissades ( $\left.n^{\circ} 31-32\right)$, le chemin d'accès moderne ( $\left.n^{\circ} 35-37\right)$, la fosse-silo de la plateforme sommitale $\left(n^{\circ} 42\right)$ et le petit fossé de l'enceinte intérieure ( $\left.n^{\circ} 3\right)$, le tout recoupé en 1904 par le réservoir du service des eaux, à droite

$\mathrm{Cl}$. Raynaud (c) CNRS

Malheureusement, aucun vestige de construction n'avait survécu à l'érosion ainsi qu'à la construction $\mathrm{du}$ réservoir moderne, ce qui interdisait une restitution de l'aménagement médiéval. L'étroitesse du sommet, probablement accentuée par l'érosion et les destructions, ne laissait de place que pour une tour, mais les deux palissades à mi-pente devaient soutenir des plateformes défensives. Le mobilier, limité à quelques fragments de céramique grise, donnait peu de prise à la datation qu'il fallait situer largement entre les XI ${ }^{e}$ et XII ${ }^{e}$ siècles ${ }^{9}$. Du moins l'hypothèse initiale d'un élément premier du castrum de Mauguio était-elle confortée : la motte appartenait bien probablement à ce moment charnière de la composition du paysage seigneurial languedocien.

Resté clos durant près d'un quart de siècle, le dossier de la motte de Mauguio s'est trouvé réactivé en 2012 à l'occasion du projet de la municipalité de réhabiliter une partie du «château » établi au pied sud de la motte, pour en faire un centre d'interprétation de l'histoire de la ville. Si aucun document n'authentifie formellement l'emplacement avant la localisation sur un plan terrier du XVIII siècle d'un " château de Maureilhan $»^{10}$, les éléments architecturaux conservés révèlent la présence en ce lieu d'une grande demeure aristocratique des XVe-XVII siècles (infra, fig. 13).

Dans un premier temps, deux sondages effectués en 2012 confirmèrent la datation ainsi que le caractère artificiel de la motte et invitèrent à étendre encore l'ampleur de ce relief castral. Aussi était-il opportun de poursuivre en 2014 les recherches dans une parcelle voisine, un jardin qui, localisé entre les deux auréoles déployées autour de la motte, permettait d'envisager l'identification du «morphogène» à l'origine de l'urbanisme circulaire du centre-ville. Si évidente qu'elle soit par son influence sur la voirie et les îlots bâtis, cette forte structure restait à déterminer dans son origine ainsi que dans sa nature : enceinte, fossé, rue, et depuis quand? 
Quatre sondages furent donc entrepris. Les sondages 1 et 2 , se développant sur 12 mètres de longueur, livrèrent l'essentiel des observations stratigraphiques en révélant la présence d'un grand fossé ayant joué le rôle de "piège sédimentaire » en conservant, au fur et à mesure de son comblement sur plus de trois mètres de profondeur, une quarantaine de couches archéologiques qui témoignent d'un long processus de transformation (fig. 10).

Fig. 10

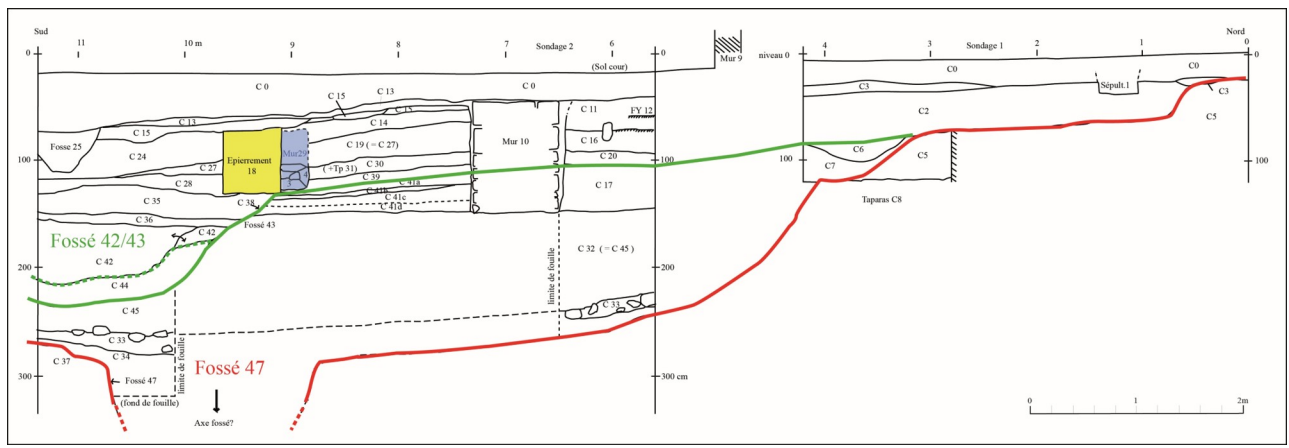

Mauguio (Hérault), coupe stratigraphique du fossé majeur dans les sondages 2014 ; les traits gras vert et rouge indiquent les deux phases principales de fonctionnement du fossé

DAO A. Lee @ Atelier d'Archéologie Lunel-Viel par une profonde excavation qui se prolonge et s'amplifie dans le sondage 2. I s'agit d'un très large fossé dont ni le fond ni la berge sud n'ont pu être atteints avant l'arrêt de la fouille à $3,30 \mathrm{~m}$ sous le sol actuel pour raison de sécurité (fig. 10, fossé 47-48, profil rouge). On peut néanmoins restituer l'ensemble du profil en prenant pour modèle la paroi nord, dégagée sur 6,5 $\mathrm{m}$ de développement. En supposant un fossé aux parois relativement symétriques en profil d'équilibre, on peut situer l'axe médian du fossé à 8 ou 9 mètres de la berge nord, ce qui donne par projection une largeur initiale de 16 à 18 mètres. Le profil de la berge nord, d'abord relativement doux, s'abaisse de $1,50 \mathrm{~m}$ pour $4 \mathrm{~m}$ avant de marquer un angle accusé qui détermine un chenal de base dont la profondeur n'est pas connue : si la fouille a dû s'interrompre à plus de 3 mètres sans atteindre le fond, l'aspect vertical de la paroi laisse envisager encore près d'un mètre jusqu'au fond du chenal, soit une profondeur minimale de $4 \mathrm{~m}$. Quelque marge 
d'erreur que l'on retienne, de l'ordre de plus ou moins $10 \%$, l'incertitude n'enlève rien à l'ampleur du fossé, ouvrage hors norme qui n'a rien à voir avec un aménagement de drainage et entre dans la classe des ouvrages défensifs.

31 La lecture stratigraphique du comblement fait apparaître une longue séquence rythmée par cinq temps forts. Si le creusement du fossé ne peut être daté précisément faute de niveaux antérieurs qui auraient fixé un terminus post-quem, le sédiment colluvial déposé au fond du chenal a livré quelques fragments de poterie des $\mathrm{XI}^{\mathrm{e}}-\mathrm{XIII}{ }^{\mathrm{e}}$ siècles (couches 33,34 ). Ce premier colmatage se poursuit et s'accentue avec un puissant niveau limono-argileux de près de 1 mètre d'épaisseur (couches 32 et 45). La texture du dépôt ainsi que la logique du ruissellement depuis un point haut adjacent, désignent la motte comme origine de ce sédiment que la présence dominante de céramique oxydante de la garrigue montpelliéraine invite à situer au premier tiers du XIII ${ }^{\mathrm{e}}$ siècle. Ce colmatage initial du fossé enregistre une première érosion de la motte, processus probablement difficile à maîtriser sur une telle masse de terre rapportée ; ce sédiment meuble et sans structure, qui plus est en position de fort relief, devait subir un ruissellement agressif lors des fortes précipitations qui marquent régulièrement le régime méditerranéen.

Dans une seconde phase, sans que l'on puisse identifier ou exclure des travaux de curage et d'entretien des rives du fossé, le colmatage l'emporte et le fossé perd une large part de son ouverture et de sa profondeur, jusqu'à ce que plusieurs niveaux de sol de terre damée ne recouvrent son comblement, toujours dans la première moitié du XIII ${ }^{e}$ siècle (sols 41a-d). Apparemment un temps délaissé, le fossé est cependant réactivé lors du creusement d'un nouveau chenal, plus étroit, moins profond et légèrement décalé vers le sud (fossé $42-43$, tracé vert). Ce fossé, d'aspect moins défensif que délimitatif, marque un troisième infléchissement de la topographie locale. À nouveau se déposent des sédiments colluviaux montrant que l'érosion de la motte se poursuit, quoique sur un mode mineur si l'on en juge à la faible puissance des dépôts : la butte serait-elle mieux protégée, son sol stabilisé aurait-il atteint un niveau d'équilibre ? Les céramiques situent ce comblement à la fin du XIII ${ }^{\mathrm{e}}$ ou au début du XIV ${ }^{\mathrm{e}}$ siècle (couches 35,44 ).

33 À nouveau et définitivement colmaté, le fossé disparaît sous les sols de terre battue d'une quatrième phase d'occupation. Celle-ci voit s'élever un premier bâtiment sur poteaux (C 30, TP 31), rapidement remplacé par un bâti à murs de terre crue, partiellement dégagé dans le sondage $2^{11}$. Au terme d'une brève occupation qui s'inscrit dans la première moitié du XIV ${ }^{e}$ siècle sans que l'on puisse préciser, le bâtiment, dont la nature et la fonction restent indéterminées, est démoli et aussitôt remplacé par une nouvelle construction aux murs de pierre et mortier de chaux qui reprend le même emplacement (épierrement 18 contre mur 29). Marqué lui aussi du sceau de la précarité, ce second édifice, tout aussi indéterminé que le précédent, est à son tour démoli et ses fondations épierrées, tandis que le sol adjacent se couvre d'un dépotoir dont le mobilier se situe une nouvelle fois avant le milieu du XIV ${ }^{e}$ siècle.

Ce quatrième temps d'occupation voit donc se succéder en une cinquantaine d'années trois états d'une construction dont les trois murs successifs, poteaux puis terre crue puis maçonnerie, marquent une profonde mutation des lieux: les fossés disparus, l'entretien et le rôle militaire de la motte semblent bien oubliés, pour des raisons que l'on évoquera plus bas. 
Le cinquième et ultime temps fort de l'occupation saisie par les sondages intervient après une longue interruption de l'occupation qui s'affirme à nouveau aux XVI $\mathrm{XVVIII}^{\mathrm{e}}$ siècles (mur 11, couches 11,13,15).

La pratique du sondage stratigraphique, seule possible face à l'exigüité du parcellaire urbain, montre là ses limites qui ne lui permettent pas de mesurer l'ampleur d'un ou de bâtiments dont la fonction reste indéterminée. Cette contrainte bride une part de l'interprétation, notamment en regard de l'interruption de l'occupation vers le milieu du XIV siècle. On ne peut en effet s'empêcher de penser à la saignée démographique de la pandémie de la fin des années 1340 , qui a pu vider de ses habitants un secteur du bourg castral, ce que les sondages suggèrent de façon ponctuelle sans en administrer la preuve; la démonstration d'une telle hypothèse nécessiterait une fouille portant sur une part significative du quartier.

\section{Topographie et évolution de la motte de Mauguio}

Représentant seulement $0,75 \%$ des trois hectares que couvre la motte, les sondages réalisés ne peuvent répondre à l'ensemble des questions posées par l'installation du castrum ainsi que par la croissance urbaine qui s'enclenche autour de ce nouveau pôle politique. L'ensemble des observations accrédite néanmoins la présence d'une butte artificielle dont on peut restituer le modelé grâce à un relevé altitudinal de plusieurs centaines de points ${ }^{12}$. Sous la végétation du jardin et les bâtiments du centre, se dessine un relief de plus de 200 mètres de diamètre pour une élévation de 18 à 20 mètres au minimum, les sondages ayant souligné la forte érosion d'une butte dont la pente à $40 \%$ représente un fragile profil d'équilibre.

Contrairement à la première approche qui laissait envisager une butte limitée par une double ligne palissadée et un fossé d'enceinte à l'altitude de $13 \mathrm{~m}$, dans le jardin municipal, celui-ci n'occupe en réalité que la partie sommitale de la motte dont le fossé majeur a été localisé en 2014 à la courbe de $5 \mathrm{~m}$. Le morphogène qui guide le tracé annulaire des rues, n'apparait donc plus désormais comme une première enceinte enserrant une basse-cour ou une première extension urbaine, mais correspond au grand fossé qui ceinture la base de la motte (fig. 11). 
Fig. 11

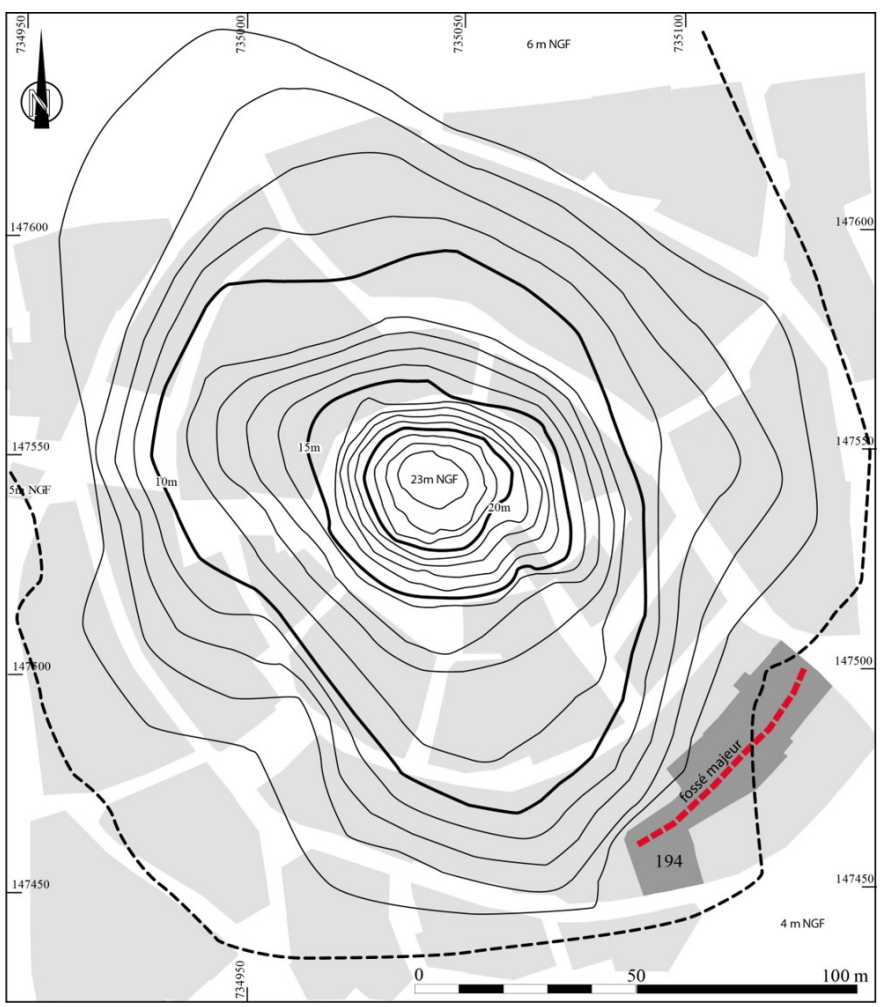

Mauguio (Hérault), restitution hypsométrique de la motte sur fond de plan des îlots bâtis (grisé) ; le fossé majeur traverse la parcelle 194 dans sa plus grande longueur, entre les courbes 5 et $6 \mathrm{~m}$ NGF relevé G. Marchand, Cl. Raynaud @ CNRS

La motte de Mauguio affecte une forme ovalaire et couvre une surface de plus de 3 ha qui en fait l'une des mottes artificielles les plus massives d'Europe. Le calcul du volume sous la pente par unité surfacique des courbes de niveau, donne une hypothèse minimale de $125000 \mathrm{~m}^{3}$ de sédiment rapporté, et une hypothèse maximale de 180000 $\mathrm{m}^{3}$ tenant compte du colmatage des abords (calcul F. Raynaud). Quelque version que l'on adopte, en tenant compte d'une masse volumique de $1500 \mathrm{~kg} \mathrm{par} \mathrm{m}^{3}$ de sédiment terreux, la masse transportée s'élève entre 190000 et 265000 tonnes de matériaux, soit l'équivalent actuel de 5000 à 7000 gros camions de travaux publics... à multiplier par deux ou trois pour tenir compte du foisonnement de la terre piochée : 10000 à 20000 camions, un sacré chantier !

De tels chiffres donnent la mesure d'une entreprise hors du commun, même à l'aune d'un chantier TGV au début du XXI ${ }^{\text {e }}$ siècle. En considérant les moyens disponibles autour de l'an Mil, pioches de fer, canastes (paniers) de bois ou de roseau portées à dos d'âne, fragiles chariots et maigres attelages de bœufs, c'est une armada d'ouvriers qui dut être réunie pour mener à bien l'entreprise, qu'il n'était probablement pas question d'étaler de longues années durant... Pas question non plus d'imaginer glaner les sédiments de curage des fossés de drainage ou de voirie, alors que s'imposait l'ouverture de plusieurs carrières à proximité du chantier.

Sans pouvoir identifier ces lieux d'extraction, qui pourraient marquer encore la topographie de la plaine, on doit cependant envisager un rapport entre l'édification de la motte et le creusement des canaux de la Roubine, de la Capouillère et du portus Melgorii castri, attesté dès le $\mathrm{XI}^{\mathrm{e}}$ siècle $^{13}$, un kilomètre au sud de la motte (fig. 12). Les 
couches de sédiment sombre hydromorphe, identifiées dans plusieurs sondages en 1989-1990, pourraient en effet provenir de ces cours d'eau canalisés. Ces chantiers ne sont cependant pas datés, de sorte que l'on ne peut établir de corrélation autre que de bon sens...

Fig. 12

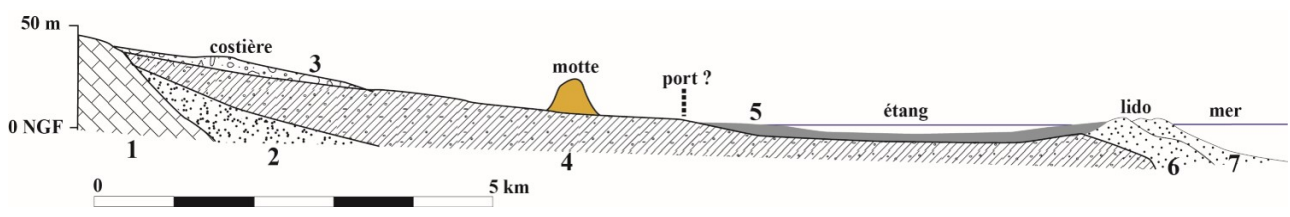

Mauguio (Hérault), coupe géomorphologique de la garrigue à la mer. 1 : affleurement du jurassique. 2 : affleurement pliocène de Saint-Aunès. 3 : formations fluviatiles, extrémité occidentale de la Costière rhodanienne. 4 : formations colluviales quaternaire ancien de la plaine. 5 : colmatage palustre de l'étang. 6 : cordon littoral ancien à cailloutis rhodaniens. 7 : cordon récent à dunes sableuses. Le portus Robine Melgorii est établi sur l'ancien rivage lagunaire, aujourd'hui colmaté

DAO Cl. Raynaud (c)

L'édification de la motte de Mauguio relève d'un chantier d'ampleur exceptionnelle.

L'origine de la main-d'œuvre et son recrutement, qui ne sont jamais mentionnés dans les textes contemporains, pose aussi question. Il semble peu convaincant, pour faire face à l'ampleur du chantier, de recourir aux corvées imposées aux tenanciers des biens seigneuriaux : deux journées d'âne ou de bœuf ici ou là, sans rapport avec la taille du chantier. On pourrait aussi envisager une main d'œuvre servile, attestée par exemple en 960 à la villa de Candillargues, près de Mauguio et participant du patrimoine comtal, où apparaissent des servis et ancillis (H.G.L., V, c. 233). Mais les corvées et les serfs pouvaient-ils suffire à la construction de la motte ? Cela reste difficile à envisager, particulièrement en Languedoc où ces pratiques restaient de portée limitée. Reste le travail rémunéré, qui pouvait compter sur les paysans des vieux centres d'habitat groupé, nombreux dans la plaine, tel celui de Lallemand (hameau de Mauguio) récemment mis au jour.

Si l'on ne peut établir de façon mécanique une relation entre la taille d'une motte et la puissance ou la richesse de son instigateur, on ne peut négliger cependant la disproportion entre l'ouvrage melgorien et la taille moyenne des mottes du premier âge féodal, dont le diamètre varie généralement entre 10 et 50 mètres pour une hauteur de 5 à 10 mètres, en restant dans le domaine des mottes artificielles. Le cas de la motte d'Olivet (Calvados), en Normandie, résume les traits de cette catégorie avec son diamètre de $10 \mathrm{~m}$, son élévation de 5 à $7 \mathrm{~m}$ assise sur un affleurement rocheux; le volume de sédiment rapporté y est évalué à $450 \mathrm{~m}^{3}$, soit $0,25 \%$ de la motte de Mauguio ! Même à Niozelles (Alpes-de-Haute-Provence), en Provence, où la motte sur relief naturel a fait l'objet d'un remblaiement évalué à $1600 \mathrm{~m}^{3}$ de sédiment, on reste à $1 \% \mathrm{du}$ volume de Mauguio ${ }^{14}$.

On pourrait multiplier les exemples mais il faudrait nécessairement tenir compte de la nature des sédiments, du substrat et particulièrement du régime des précipitations, qui constitue une sévère menace pour l'érosion de la motte. En effet, il ne paraît guère convaincant de comparer des buttes artificielles de régions à climat tempéré aux précipitations étales, favorables à un couvert végétal protecteur, avec des mottes en climat méditerranéen semi-aride une partie de l'année puis soumis à de violentes précipitations: au demeurant, les mottes artificielles restent rares en milieu 
méditerranéen et couvrent des surfaces de 0,06 à 0,1 hectare, soit 2 à $2,5 \%$ de la motte de Mauguio.

C'est en revanche un relief " mixte », site rocheux surélevé artificiellement, qu'occupe la tour de Teulet, dans l'arrière-pays de Béziers, sur un mamelon qui ne dépasse guère la hauteur de $2,5 \mathrm{~m}$ pour une quarantaine de mètres de diamètre à la base $\mathrm{e}^{15}$. Le podium sur lequel s'élève la tour, est défini par un fossé large de $6 \mathrm{~m}$ et profond de $2 \mathrm{~m}$ environ. Relevons une morphologie comparable pour les tours de Savoie-Vieille ou de Caux (Vendres, Mèze ; Hérault); ces «roques » de l'aristocratie biterroise restent bien en deçà du gigantisme melgorien.

En Languedoc, les mottes de quelque ampleur occupent en réalité des collines naturelles remodelées, d'une vingtaine de mètres de diamètre pour une hauteur de 10 mètres, à l'exemple de la motte du Mourrel à Molleville (Aude), ou plus massive, de Puy-Redon à Laurac (Aude). À propos de ces «mottes naturelles ", que le terme roque définit mieux et distingue des mottes artificielles, on peut dresser le même constat que précédemment sur la nécessité de comparaisons régionales pour lesquelles s'offrent de multiples cas d'étude, à commencer par les castra voisins de Sommières, Vauvert et bien sûr Montpellier. Ce faisant, on doit aussi s'interroger sur les sites castraux dépourvus de motte comme c'est le cas à Lunel (Hérault) où l'emprise castrale, bien marquée sur le plan parcellaire avec un diamètre d'une centaine de mètres, ne s'élève pas au-dessus de la platitude de la ville, ou encore à Lattes où le castrum ne semble pas non plus s'ériger en hauteur ${ }^{16}$.

\section{6. Émergence, puissance et déchéance : de la motte au château}

47 L'analyse géographique souligne l'absence de facteur naturel qui aurait pu guider le choix du site pour l'installation du castrum Melgorium : ni la topographie ni le terroir n'y sont meilleurs qu'en d'autres points de la plaine. Quelques kilomètres au nord, plusieurs piochs culminent entre 50 et $60 \mathrm{~m}$ et offraient, moyennant quelques aménagements, d'excellents sites défensifs : ils ont été négligés et ne semblent jamais occupés. C'est au contraire un lieu atypique, émergeant de quelques mètres seulement au-dessus de l'étang, qui a retenu le choix comtal.

48 À l'évidence, il importait de s'assurer une position centrale dans la plaine et près du littoral, de voir et d'être vu au loin. Mais plus encore, cette plaine offrait une zone de peuplement densément structurée depuis la période romaine. En effet, les vestiges de limitatio antique révèlent une campagne organisée et exploitée, et plusieurs établissements s'imposent dans la campagne autour du site de la motte dans un rayon de 3 à 5 kilomètres. C'est le cas de plusieurs chefs-lieux de paroisses où les prospections archéologiques révèlent un fort legs de l'Antiquité tardive. D'autres établissements, plus mal perçus de prime abord, se trouvent éclairés par des fouilles récentes qui révèlent une exploitation dynamique dès le haut Moyen Âge, comme l'habitat de Lallemand, récemment fouillé17. Par ailleurs le camin salinié, au bord duquel la motte est établie, constitue un itinéraire hérité de l'Antiquité dont la vitalité ne se démentit pas jusqu'à la période moderne. 

fouillé...) qui reçoit alors des constructions traduisant peut-être une phase d'urbanisation. Mettra-t-on cette observation au compte de l'abandon de la première forteresse de terre et de bois, désormais inadaptée à la fois aux nouvelles techniques guerrières et au cadre de vie princier? Ce tournant correspond peut-être en effet à l'acquisition de la seigneurie de Mauguio par les comtes de Toulouse grâce au mariage de Raymond VI en 1172. On sait que dans les premières années du XIII ${ }^{e}$ siècle, le toulousain fit bâtir un palais pour marquer son emprise ${ }^{18}$. À cette occasion, un texte mentionne l'achat d'une maison confrontant le château de Mauguio et sa tour, ainsi que deux autres maisons (stari), ce qui donne une idée de la densité des constructions autour de la motte. Les sondages n'ont semble-t-il livré aucun élément de la nouvelle résidence comtale, de sorte que l'on se gardera d'extrapoler à partir de cette donnée textuelle.

\section{De la motte au bourg : le castrum du bas Moyen Âge}

L'urbanisme moderne de la ville de Mauguio déborde largement les enceintes circulaires autour de la motte et s'inscrit dans un quadrilatère délimité par de larges boulevards qui occupent d'anciens fossés, mentionnés sur le plan cadastral de 1818 et toujours prégnants dans le tissu actuel (fig. 6). Dans ce périmètre de près de treize hectares, le bourg s'est maintenu jusqu'au milieu du XXe siècle. Encore visibles dans les années 1950 et partiellement en eau lors de fortes pluies, les fossés encadrant le centre ancien représentaient le seul vestige de la grande enceinte polygonale abattue à l'issue du siège de la ville par Louis XIII en 1622. La date de construction de ces fortifications est par contre très incertaine. Des «douves et fossés de l'entour des murailles de la ville » sont bien mentionnés à la fin du XIII ${ }^{e}$ siècle ${ }^{19}$ mais il est difficile de dire si cela concerne la grande enceinte ou l'enceinte circulaire de la motte, le texte ne fournissant aucun indice topographique.

Quand et comment passe-t-on de l'habitat resserré autour de la motte et protégé par une enceinte circulaire, à la formation de nouveaux quartiers nécessitant une plus vaste enceinte? Quelques éléments architecturaux témoignent d'une extension ancienne, particulièrement vers le nord, autour de la porte de Peyrefiche où subsiste un bel ensemble bâti des XIII ${ }^{\mathrm{e}}$-XIV ${ }^{\mathrm{e}}$ siècles. 

série d'observations décisives quant à l'analyse de la genèse du bourg castral. Si les données manquent encore pour dater la création de la motte, sa topographie et son extension sont désormais cernées grâce à la localisation d'un ample fossé, probablement l'enceinte majeure du premier castrum qui s'organise sur et autour d'une motte artificielle d'une ampleur exceptionnelle. l'accumulation des sédiments d'érosion d'une motte qui ne semble plus entretenue, peut-être dès le XII ${ }^{e}$ ou le début du XIII ${ }^{e}$ siècle. La prise de contrôle de la seigneurie par les comtes de Toulouse, au dernier tiers du XII ${ }^{e}$ siècle, semble préluder à une phase de réaménagement et de construction au pied d'une motte déclassée, tant au niveau militaire qu'à l'égard de son exiguïté qui réduisait son " habitabilité ». Ce processus se trouve confirmé et amplifié avec le remblaiement final du fossé au tournant des XIII et $\mathrm{XIV}^{\mathrm{e}}$ siècles, qui voit s'élever de nouvelles constructions.

Si l'évolution nous échappe ensuite, faute de données stratigraphiques, les éléments d'une architecture aristocratique des $\mathrm{XV}^{\mathrm{e}} \mathrm{XVII}{ }^{\mathrm{e}}$ siècles, conservés dans plusieurs bâtiments modernes, imposent l'image d'un château-résidence. Ces bâtiments, partiellement acquis par la ville, font l'objet d'un programme de réhabilitation et 
d'installation d'un centre d'interprétation de l'histoire de la ville. Les résultats de notre enquête archéologique trouveront à s'exprimer dans ce cadre (fig. 13).

Fig. 13

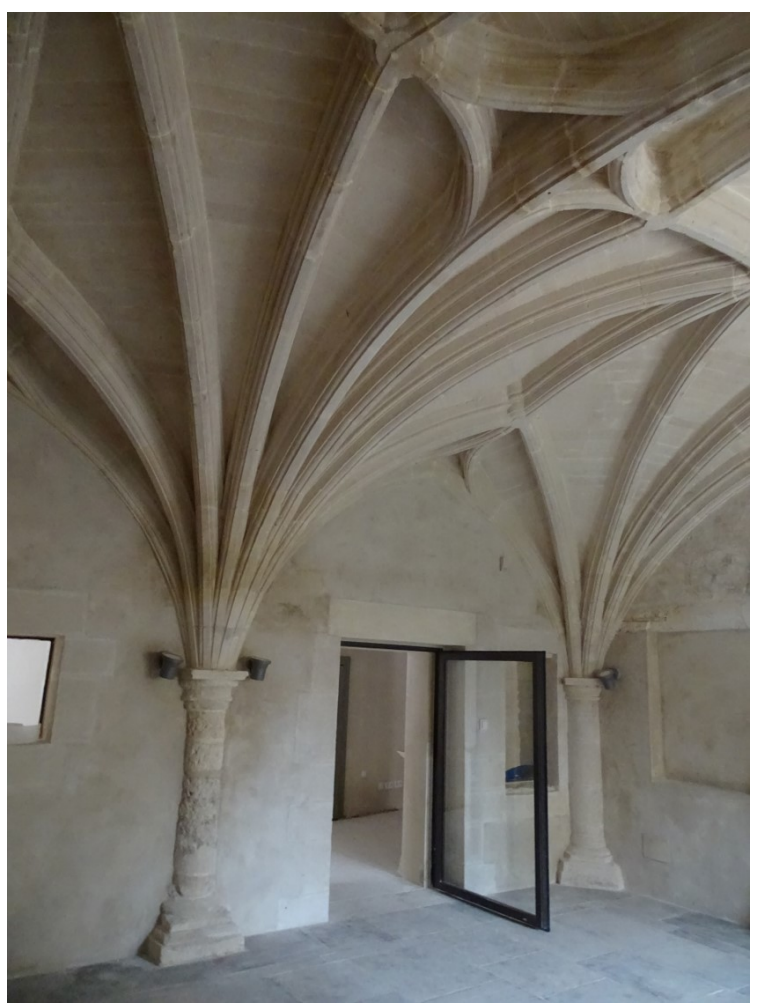

Mauguio (Hérault), la salle gothique du château

(C) Mauguio Carnon

\section{BIBLIOGRAPHIE}

BERTHELÉ, Joseph. Éclaircissements topographiques. Archives de la ville de Montpellier, V, 1928, p. 3-16.

BONNET, Émile. Antiquités et Monuments du département de l'Hérault. Montpellier : impr. de Ricard Frères, 1905.

CARBONNEL, Yves et al. De l'archéologie romantique à la prospection systématique. In BARRAL I ALTET, Xavier (édit.). Le paysage monumental de la France autour de l'An Mil. Paris : Picard, 1987.

MACÉ, Laurent. Les comtes de Toulouse et leur entourage. Rivalités, alliances et jeux de pouvoir, XII ${ }^{e}-X I I I^{e}$ siècles. Toulouse : Privat, 2000.

MOUTON, Daniel. Mottes castrales en Provence. Les origines de la fortification privée au Moyen Age, Documents d'Archéologie Française, vol. 102, Paris, 2008, 151 p. 
PARODI, Anne. La plaine du Languedoc oriental au haut Moyen Âge : textes et archéologie de l'espace rural. Thèse de doctorat, Université Paris I Panthéon-Sorbonne, 1992, 3 vol.

PLATTER, Félix et Thomas. Félix et Thomas Platter à Montpellier. Notes de voyage de deux étudiants bâlois. Montpellier : C. Coulet, 1892.

RAYNAUD, Claude, FAVORY, François, PARODI, Anne, et al. Recherches sur les cantons de Lunel et Mauguio, bilan 1990, Archéologie en Languedoc, 17, 1993, p. 87-124.

RAYNAUD, Claude. Du castrum à la ville : Lunel au Moyen Age, actes du colloque international Des Tibbonides à Maïmonide : rayonnement des Juifs andalous en pays d'Oc médiéval, Montpellier, décembre 2004. Paris : éditions du Cerf, 2009, p. 35-59.

SCHNEIDER, Laurent. Une vicaria languedocienne du $\mathrm{X}^{\mathrm{e}}$ siècle : Popian en Biterrois, Annales $d u$ Midi, 109, 1997, p. 401-442.

TARROU, Liliane. D'un monde à l'autre. L'émergence d'un établissement agricole, du VIII ${ }^{\mathrm{e}}$ au XI siècle : le site de Lallemand à Mauguio. In [exposition, Lattes, musée Henri Prades, 2017-2018] Circulez, y'a tout à voir. Gand : Snoeck, 2017, p. 64.

\section{NOTES}

1. - PLATTER, p. 366.

2. - BONNET, p. 564 ; BERTHELÉ, p. 3-16.

3. - CARBONNEL, p. 401.

4. - THÉRON, René. L'Écho du Vidourle, années 1970, AD Hérault, PAR 604, PAR 113, nº 6es chroniques villageoises rassemblent un riche fond de traditions orales recueillies dans les années 1930-1960.

5. - PARODI, RAYNAUD, 1993, p. 87-124. Les sondages de 1989-1990 ont été réalisés par Claude Raynaud avec Anne Parodi. Les sondages de 2012 ont bénéficié de la collaboration d'A. Garnotel (UMR 5140).

6. - Cartulaire de Gellone, p. 56.

7. - Carte des pêcheries de l'étang au XIV ${ }^{\mathrm{e}}$ siècle. (AD Hérault, G 2046-1).

8. - La représentation des bâtiments sur la carte repose sur les conventions habituelles des joints larges entre les pierres, et les tuiles arrondies couvrant les joints sur les toitures. De façon surprenante, le dessin médiéval révèle certaines similitudes avec le belvédère bâti au début du $\mathrm{XX}^{\mathrm{e}}$ siècle au sommet de la motte (infra, fig. 4), ressemblance peut-être attribuable à des échanges entre Joseph Berthelé, qui ne pouvait ignorer l'existence de la carte du XIV siècle, et la municipalité ou l'architecte de Mauguio... Cette circonstance, qui trouverait peut-être confirmation au travers des registres de délibérations municipales, enrichit encore la densité patrimoniale de la motte de Mauguio.

9. - PARODI, Anne. In RAYNAUD, 1993, p. 120-121.

10. - AD Hérault, $G$ 4443. Les mentions d'archives sont dues aux recherches de M. Manilève.

11. - Mur 29 : technique de la bauge identifiée par J.-Cl. Roux, archéologue de la construction en terre (UMR 5140).

12. - Ce relevé a été réalisé en 1990 sous la direction de Georges Marchand (1951-2014), ingénieur topographe et archéologue rattaché à l'UMR 5140, Archéologie des Sociétés Méditerranéennes.

13. - Cart. Maguelone, I, p. 9

14. - MOUTON.

15. - SCHNEIDER. 
16. - RAYNAUD, 2009, p. 35-59. Les bourgs castraux de Sommières et Posquières-Vauvert sont en cours d'étude.

17. - TARROU, p. 64.

18. - MACÉ, p. 48.

19. - AD Hérault, $G 2185$.

20. - AD Hérault, $2^{\mathrm{E}} 95,506$ : vue 3 ; notaire Junin.

\section{RÉSUMÉS}

Le bourg médiéval de Mauguio s'est fixé puis développé autour d'un château à motte établi par le lignage comtal des Melgueil, à la charnière des $\mathrm{X}^{\mathrm{e}}-\mathrm{XI}^{\mathrm{e}}$ siècles. Après avoir dicté les lignes de force de l'urbanisme médiéval, la motte s'est trouvée reléguée au second plan, sous l'effet de l'évolution de la société seigneuriale, de la croissance du bourg ainsi que des transformations du cadre urbain. Jamais mentionnée avant la fin $\mathrm{du} \mathrm{XVI}^{\mathrm{e}}$ siècle, longtemps négligée par les historiens, la motte de Mauguio a cependant survécu à une érosion séculaire, accueillant successivement un moulin à vent, un château d'eau puis un jardin public. Depuis les années 1990, le programme municipal de revalorisation du centre médiéval a permis de réaliser une série de sondages archéologiques et de repérages topographiques qui ramènent au grand jour un monument majeur du patrimoine languedocien et cernent les temps fort de l'histoire du castrum. $\mathrm{Du}$ haut de ses 200000 tonnes de terre rapportée, la motte de Mauguio s'impose désormais comme l'une des plus importantes buttes artificielles de l'Europe médiévale.

The mediaeval market town of Mauguio grew up and expanded around a motte-and-bailey castle built by the Counts of Melgueil, on the cusp of the $10^{\text {th }}$ and $11^{\text {th }}$ centuries. The motte laid down the major outlines of mediaeval town planning before finding itself relegated to second place, under the effect of changes in feudal society, the growth of the town and transformations in the urban context. Never mentioned before the end of the $16^{\text {th }}$ century and long neglected by historians, the Mauguio motte nevertheless survived secular erosion and has been home to a windmill, a water tower and a public garden in succession. Since the 1990s, the municipal rehabilitation programme for the mediaeval centre has included a series of archaeological and topographical surveys, thereby reinstating clearly a major monument of the Languedoc heritage and identifying the highlights of the history of the castrum. From the top of its 200,000 tonnes of brought-in earth, the Mauguio motte is now established as one of the most important artificial mounds in mediaeval Europe.

\section{INDEX}

Index géographique : Mauguio, Villeneuve-les-Maguelone, Hérault

Mots-clés : motte, château, genèse urbaine, enceinte, Melgueil

Keywords : motte, castle, urban genesis, enclosure, Melgueil 
AUTEUR

CLAUDE RAYNAUD

CNRS-Université Montpellier III, Archéologie des Sociétés Méditerranéennes 